Canadian

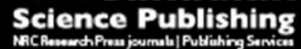

Canadian Journal of Physiology and Pharmacology Revue canadienne de physiologie et pharmacologie

\title{
Stanniocalcin 2 induces oxaliplatin resistance in colorectal cancer cells by upregulating P-glycoprotein
}

\begin{tabular}{|r|l|}
\hline Journal: & Canadian Journal of Physiology and Pharmacology \\
\hline Manuscript ID & cjpp-2015-0530.R1 \\
\hline Danuscript Type: & Article \\
\hline Complete List of Authors: & $\begin{aligned} \text { Yuan, Qiong; Wuhan University of Science and Technology, Department of } \\
\text { Pharmacology, Medical College } \\
\text { Zhan, Lin; Wuhan University of Science and Technology, Department of } \\
\text { Pharmacology, Medical College } \\
\text { Zhang, Li-Li; Wuhan University of Science and Technology, Department of } \\
\text { Histology and Embryology, Medical College } \\
\text { Wang, Qiang; Medical College, Wuhan University of Science and } \\
\text { Technology } \\
\text { Liu, Juan; Wuhan University of Science and Technology, Department of } \\
\text { Pharmacology, Medical College } \\
\text { Jiang, Zhen-Yu; Wuhan University of Science and Technology, Department } \\
\text { of Pharmacology, Medical College } \\
\text { Hu, Xia-Min; Wuhan University of Science and Technology, Department of } \\
\text { Pharmacology, Medical College } \\
\text { Yuan, Xin-Chu; Wuhan University of Science and Technology, Department } \\
\text { of Histology and Embryology, Medical College }\end{aligned}$ \\
\hline Keyword: & $\begin{array}{l}\text { multidrug resistance, stanniocalcin 2, P-glycoprotein, phosphoinositide 3- } \\
\text { kinase/Akt, oxaliplatin }\end{array}$ \\
\hline
\end{tabular}




\title{
Stanniocalcin 2 induces oxaliplatin resistance in colorectal cancer
} cells by upregulating P-glycoprotein

\author{
Qiong Yuan ${ }^{1,3+}$, Lin Zhan ${ }^{1+}$, Li-Li Zhang ${ }^{2}$, Qiang Wang ${ }^{4}$, Juan Liu ${ }^{1}$, Zhen-Yu Jiang ${ }^{1}$, \\ Xia-Min $\mathrm{Hu}^{1,3}$, Xin-Chu Yuan ${ }^{2 \dagger}$ \\ ${ }^{1}$ Department of Pharmacology, Medical College, Wuhan University of Science and \\ Technology, Wuhan 430065, China
}

${ }^{2}$ Department of Histology and Embryology, Medical College, Wuhan University of Science and Technology, Wuhan 430065, China

${ }^{3}$ Drug Research Base of Cardiovascular and Cerebral Vascular, Medical College, Wuhan University of Science and Technology, Wuhan 430065, China

${ }^{4}$ Department of Immunology, Medical College, Wuhan University of Science and Technology, Wuhan 430065, China

*Correspondence and request for reprints to Professor Xin-Chu Yuan

Department of Histology and Embryology, Medical College, Wuhan University of Science and Technology, Heping Road 947\#, Qingshan District, Wuhan, Hubei Province, P.R. China

Tel/Fax: +86 27 68893280; Email: y5307@126.com

${ }^{+}$These authors contributed equally to this work. 


\begin{abstract}
Multidrug resistance (MDR) limits the anticancer effects of chemotherapy in patients with metastatic colorectal cancer (CRC). Oxaliplatin is a common component of combinational therapeutic regimens administered to patients with metastatic CRC; however, it is also used as a constituent of adjuvant therapy for patients at a risk of recurrent disease. In the present study, we investigated the role of stanniocalcin 2 (STC2) in chemoresistance. STC2 knockdown sensitized chemoresistant CRC cells to oxaliplatin. Moreover, the expression of exogenous STC2 in chemonaïve CRC cells induced oxaliplatin resistance. We confirmed that STC2 upregulated P-glycoprotein (P-gp) expression in CRC cells. Furthermore, shRNA against phosphoinositide 3-kinase (PI3K) or Akt inhibited the action of STC2 on P-gp upregulation and MDR in CRC. To our knowledge, this is the first report to demonstrate the induction of oxaliplatin resistance in CRC cells in response to STC2 stimulation of P-gp via the PI3K/Akt signaling pathway.
\end{abstract}

Keywords: multidrug resistance; stanniocalcin 2; P-glycoprotein; phosphoinositide 3-kinase/Akt; oxaliplatin 


\section{Introduction}

Chemotherapy is the standard treatment for patients with metastatic colorectal cancer (CRC); however, most patients ultimately develop drug resistance. Oxaliplatin is an efficacious third-generation platinum-based compound that is commonly used to treat CRC (Meyerhardt and Mayer 2005). This result supports the clinical significance of oxaliplatin-containing regimens in CRC management. Nevertheless, despite this demonstrated efficacy, virtually all metastatic CRC eventually become resistant to oxaliplatin, with a median disease progression period of 8 months (Goldberg et al. 2004). However, the mechanism underlying drug resistance to oxaliplatin remains unknown.

Stanniocalcin (STC), which comprised STC1 and STC2, is a glycoprotein hormone that was originally discovered in fish and has been implicated in calcium and phosphate homeostasis (Wagner et al. 1998). In mammals, STC2 is widely expressed in both developing and adult tissues (Law and Wong 2010; 2013; Jepsen et al. 2015). Recently, researchers have suggested the involvement of STC2 in the progression of many types of cancer (Hashemzadeh et al. 2014). Higher STC2 expression levels have been detected in gastric cancers relative to normal mucosa and have also been closely associated with lymph node metastasis and venous tumor invasion (Yokobori et al. 2010). STC2 secretion from colon cancer cells has been confirmed in a simulated model of the tumor microenvironment (Zeng et al. 2013), suggesting an important role of STC2 in the development and progression of colon cancer. Despite these pioneering studies, the clinical significance and molecular mechanism of STC2 in 
cancers still remain controversial and their further elucidation is warranted. Moreover, the association of STC2 with oxaliplatin resistance in colon cancer has not yet been investigated; therefore, we speculated that STC2 regulates oxaliplatin-induced multidrug resistance (MDR) in CRC.

P-glycoprotein (P-gp), a 170-kDa protein encoded by the human multidrug-resistance $1(M D R 1)$ gene, belongs to the ATP-binding cassette (ABC) superfamily of membrane transporters (Loo and Clarke 2005). Among these membrane transporters, P-gp plays the most important role in reducing the intracellular accumulation of anticancer drugs (Gottesman and Ling 2006). The P-gp protein is found in high concentrations at the apical surfaces of colonic epithelial cells and is also known to mediate drug resistance in CRC (Kuan et al. 2011). Researchers have confirmed that in certain tumors, microenvironmental factors including hypoxia can induce P-gp upregulation as part of a pathway that functions during resistance to chemotherapeutics (Comerford et al. 2002). Various chemically unrelated anticancer drugs, including paclitaxel, doxorubicin, and vinblastine, are P-gp substrates (Szakacs et al. 2006). Recently, oxaliplatin was also reported to upregulate P-gp expression in drug-resistant CRC cells (Wang et al. 2015).

Cancer signaling pathways associated with MDR including the phosphoinositide 3-kinase (PI3K)/Akt pathway, are known to play pivotal roles in the regulation of drug-induced functional activities such as DNA damage-induced apoptosis, inhibition of cell growth, and anti-metastatic progression (Persons et al. 1999; Wang et al. 2000). PI3K/Akt influenced the development of MDR in colon cancer (Wang et al. 2014). 
Recent evidence has emphasized the interplay between PI3K/Akt signaling and P-gp-mediated MDR in CRC (Jiang and Liu 2008; Kuo et al. 2011). Secreted STC1 promoted the metastatic potential of breast cancer cells via PI3K/Akt activation (Murai et al., 2014). However, the effect of STC2 on PI3K/Akt signaling pathway activation in the context of MDR colon cancer remains unknown.

In the present study, we hypothesized that STC2 regulates PI3K/Akt signaling pathway and therefore oxaliplatin-induced and P-gp-mediated MDR in colon cancer.

\section{Materials and Methods}

Cell lines and the in vitro chemoresistance model

The human CRC cell lines HT29, RKO, and HCT116 were obtained from the American Type Culture Collection (ATCC, Manassas, VA, USA). The oxaliplatin-resistant cell lines HT29-OxR, RKO-OxR, and HCT116-OxR were developed as previously described (Yang et al. 2006). Oxaliplatin-resistant cells were continuously cultured in $2 \mathrm{mM}$ oxaliplatin (Sigma, St. Louis, MO, USA) unless otherwise indicated. HCT116 cells were exposed to STC2 $(10 \mathrm{pg} / \mathrm{ml}$, USCN Life Science, Wuhan, China) in DMEM (Hyclone, Utah, USA) supplemented with $10 \%$ FBS (Hyclone) for $24 \mathrm{~h}$. The treated cells were subsequently grown to $80 \%$ confluence and were used at the $12^{\text {th }}$ passage from the initial STC2 treatment.

\section{STC2 ELISA assay}

The level of STC2 in cell culture was determined using a human STC2 ELISA kit (USCN Life Science) according to the manufacturer's instructions.

Plasmid transfection 
HCT116-OxR cells were transfected separately with the recombinant plasmids pcDNA3.1-N-shRNA, pcDNA3.1-STC2-shRNA (Shanghai Genechem Company, Shanghai, P.R. China), pcDNA3.1-PI3K-shRNA, and AKT-shRNA (Santa Cruz Biotechnology, CA, USA) using the FuGENE HD reagent (Roche, Basel, Switzerland) according to the manufacturer's instructions. The cells were used for experiments $48 \mathrm{~h}$ after transfection.

Western blot analysis

The expression levels of several proteins were detected by Western blot analysis. Proteins were separated using $10 \%$ sodium dodecyl sulfate-polyacrylamide gel electrophoresis and transferred onto a polyvinylidene fluoride (PVDF) membrane at $100 \mathrm{~V}$ for $1 \mathrm{~h}$. Subsequently, the membrane was incubated in TBS/T buffer $[20 \mathrm{mM}$ Tris- $\mathrm{HCl}(\mathrm{pH} 7.6), 150 \mathrm{mM} \mathrm{NaCl}, 0.1 \%$ Tween-20] containing 5\% non-fat milk at room temperature for $2 \mathrm{~h}$. Specific primary antibodies including rabbit anti-human STC2 (diluted 1:500), rabbit anti-human P-gp (diluted 1:1000), rabbit anti-human PI3K (diluted 1:1000), rabbit anti-human p-Akt (diluted 1:1000), and mouse anti-human GAPDH (diluted 1:2000) were from Santa Cruz Biotechnology. All antibodies were diluted in TBST buffer [50 mM Tris- $\mathrm{HCl}(\mathrm{pH} 7.4), 150 \mathrm{mM} \mathrm{NaCl}$, $0.1 \%$ Tween-20] with which the PVDF membranes were incubated at $4^{\circ} \mathrm{C}$ overnight. Subsequently, the PVDF membranes were incubated with the corresponding horseradish peroxidase-conjugated secondary antibodies for $60 \mathrm{~min}$ at room temperature. Signal detection was performed using an enhanced chemiluminescence reagent (Amersham Biosciences, Piscataway, NJ, USA). 


\section{Apoptosis determination}

Annexin V-propidium iodide (PI) staining (Beyotime Biotechnology, Jiangsu China) was analyzed using flow cytometry. Cells were collected and washed twice with phosphate-buffered saline (PBS), followed by resuspension in $250 \mu$ l binding buffer. Five microliter FITC-annexin V and $10 \mu 1 \mathrm{PI}(20 \mu \mathrm{g} / \mathrm{ml})$ were added to each $100-\mu 1$ cell suspension, and the cells were incubated at room temperature for $15 \mathrm{~min}$. Subsequently, $400 \mu \mathrm{PBS}$ was added to the cell suspensions and the samples were analyzed by flow cytometry (Becton-Dickinson, Franklin Lakes, NJ, USA).

To measure caspase-3 activity, cells were seeded into 12-well plates at a density of $1 \times 10^{5}$ cells/well, and caspase-3-like protease activity in cell lysates was measured using a colorimetric caspase-3 assay (Beyotime Biotechnology). Caspase- 3 activity is expressed in values of optical density at $405 \mathrm{~nm}\left(\mathrm{OD}_{405}\right)$.

Detection of P-gp function

Rhodamine-123 efflux was used to detect the P-gp function of cells. Cells were cultured in 6-well plates; at 70-80\% confluence, rhodamine-123 (Sigma) was added to the cells at a final concentration of $0.25 \mu \mathrm{g} / \mathrm{ml}$ and incubated at $37^{\circ} \mathrm{C}$ for $1 \mathrm{~h}$. Cells were washed 3 times with PBS at $4^{\circ} \mathrm{C}$ and re-suspended at $5-10 \times 10^{5}$ cells $/ \mathrm{ml}$ in PBS. Rhodamine-123 fluorescence was analyzed using a FACStar flow cytometer (BD Biosciences, CA, USA) equipped with an argon laser. The blast population was gated by forward and side scatter characteristics. Rhodamine-123 fluorescence of 10,000 cells was measured logarithmically through a 530-nm bandpass filter at an excitation wavelength of $488 \mathrm{~nm}$. Cells that were not incubated with rhodamine-123 served as a 
negative control. Rhodamine-123 efflux was measured by counting cells in the M1 region of the plot and was expressed as a percentage. The higher the percentage of cells in the M1 region, the greater the cellular rhodamine-123 efflux and P-gp function.

Transepithelial transport of rhodamine-123

Transepithelial electrical resistance (TEER) of CRC-OxR cell monolayers cultured on a transwell chamber was monitored $24 \mathrm{~h}$ prior to transport studies using a Millicell ERS testing device (Millipore, Bedford, MA). The monolayers with a TEER of more than $250 \Omega \cdot \mathrm{cm}^{2}$ were used for transport studies. Prior to the addition of rhodamine-123, the monolayers were rinsed twice and then incubated in Hanks' balanced salt solution containing $25 \mathrm{mM}$ HEPES and $25 \mathrm{mM}$ glucose (HBSS buffer, $\mathrm{pH}$ 7.4). Rhodamine- 123 was placed either on the apical $(1.5 \mathrm{ml})$ side at a concentration of $5 \mu \mathrm{M}$ and measured on the opposite side to the placed site at $37{ }^{\circ} \mathrm{C}$ periodically for $120 \mathrm{~min}$. The transepithelial transport of rhodamine-123 across cell monolayers was determined fluorometrically using the Shimadzu fluorescence spectrophotometer RF-5000 (Tokyo, Japan).

Statistical analysis

Data are presented as the mean \pm standard error of the mean (SEM). An independent sample $t$ test was used to assess significant differences among continuous variables. A one-way analysis of variance followed by Tukey's test was used for multiple comparisons. All calculations were performed using the Statistical Package for the Social Sciences (SPSS) software, version 11.0 (SPSS Inc., Chicago, IL, USA). 
Differences with $P$ values of $<0.05$ were considered to be statistically significant.

\section{Results}

STC2 is overexpressed in oxaliplatin-resistant CRC cells

We compared STC2 protein expression and secretion profiles of the three parental (chemonaïve) CRC cell lines HT29, HCT116, and RKO with those of their oxaliplatin-resistant derivative cells. STC2 expression was elevated in all three oxaliplatin-resistant cell lines (Fig. 1A). We also detected elevated STC2 secretion in all three chemoresistant cell lines compared with that seen in the parental cells (Fig. 1B). Because all three oxaliplatin-resistant CRC cell lines secreted greater amounts of STC2, we hypothesized that secreted STC2 plays a role in the development of acquired resistance to oxaliplatin in CRC cells.

STC2 induces oxaliplatin resistance in CRC cells

To confirm the functional role of STC2 in the induction of chemoresistance, the levels of STC2 in parental HCT116-OxR and HCT116 cells were altered using STC2-shRNA and exogenous STC2 protein, respectively. We successfully silenced STC2 expression and secretion (Fig. 2A-B). Decreased STC2 expression resulted in a dose-dependent increase in the sensitivity of HCT116-OxR cells to oxaliplatin treatment (Fig. 2C-D). We also detected the effect of STC2-shRNA on the survival of chemonaïve HCT116 cells (supplementary materials). Western blot analysis of STC2 expression in HCT116 cells (Fig. S1A-B) showed that STC2-shRNA had no effect on 
the sensitivity to oxaliplatin treatment (Fig. S1C-D). Conversely, treatment with recombinant STC2 protein $(10 \mathrm{pg} / \mathrm{ml})$ reduced both the apoptosis frequency and caspase-3 activity (Fig. 3A-B).

Correlation between P-gp and STC2 in oxaliplatin-resistant cells

During the development of chemoresistance to oxaliplatin, cancer cells likely acquire an enhanced ability to transport the drug outside the cells; this results in the suppression of chemotherapeutic cytotoxicity. To confirm the relationship between P-gp and STC2 with regard to chemoresistance induction in CRC, we first analyzed P-gp protein expression in three parental CRC-OxR cell lines, namely HT29-OxR, HCT116-OxR, and RKO-OxR. P-gp was upregulated in all three CRC-OxR cell lines (Fig. 4A). To further confirm the regulatory effect of STC2 on P-gp expression, cells were treated with STC2-shRNA. Compared with N-shRNA, STC2-shRNA significantly downregulated P-gp expression in the HCT116-OxR line (Fig. 4B). The function of P-gp was detected by the concentration and apical to basolateral transport of rhodamine-123. As shown in Fig. 4C and D, STC2-shRNA reversed the P-gp effect on drug efflux and on the apical to basolateral transport $(P<0.01)$.

PI3K/Akt mediates the effect of STC2 on P-gp

To confirm the role of PI3K/Akt in STC2-induced MDR in CRC, we first evaluated PI3K and phospho-Akt (p-Akt) expression. As shown in Fig. 5A, both PI3K and p-Akt were significantly upregulated in the HCT116-OxR, HT-29-OxR, and 
RKO-OxR cell lines. Furthermore, to confirm that STC2 upregulated P-gp protein expression and increased drug efflux, we used shRNA against both PI3K and Akt. Compared with N-shRNA, P-gp protein expression was significantly downregulated following treatment with PI3K-shRNA or AKT-shRNA in HCT116-OxR cells (Fig. 5B, $P<0.05)$. Additionally, compared with N-shRNA, both PI3K-shRNA and AKT-shRNA increased the concentration of rhodamine-123 in HCT116-OxR cells (Fig. 5C, $P<0.01$ ). Furthermore, PI3K-shRNA and AKT-shRNA reversed the function of P-gp, as shown by a decrease in the apical to basolateral transport of rhodamine-123 (Fig. 5D, $P<0.01$ ). These results indicate that blockade of the PI3K/Akt signaling pathway alleviated the effect of STC2 on chemoresistance to oxaliplatin in HCT116 cells via P-gp, which promotes oxaliplatin efflux.

\section{Discussion}

Metastatic CRC leads to the death of 50,000 patients annually in the USA because of primary or acquired resistance to chemotherapy (Davies and Goldberg 2008). Therefore, it is critical to delineate drug resistance mechanisms to commonly used therapeutic agents, such as oxaliplatin, to improve the survival of patients with CRC. The mechanisms underlying the cytotoxic effects of oxaliplatin have not yet been completely elucidated. The present study established a link between STC2 and oxaliplatin resistance. This link possibly involves a P-gp-mediated mechanism. First, we showed that only STC2 was commonly overexpressed in three CRC cell lines with acquired resistance to oxaliplatin. Second, we showed that STC2 suppression 
sensitized resistant CRC cells to oxaliplatin and that STC2 overexpression protected chemonaïve CRC cells from oxaliplatin-related cell death. Third, we demonstrated that STC2 regulated the membrane transporter P-gp by activating the P13K/Akt signaling pathway.

STC is a calcium-regulating hormone that was first identified in bony fishes and is known to be released into the bloodstream to regulate $\mathrm{Ca}^{2+}$ and phosphate uptake in different target organs (Wagner and Jaworski 1994). The human STC2 gene, which encodes a 302-amino acid protein, is a glycoprotein hormone that is primarily expressed in the kidneys, heart, pancreas, and spleen (Chang et al. 2003). STC2 is internalized and interacts with Ran-binding $M$ protein to transactivate the liganded androgen receptor (Shin and Sohn 2014). Previous studies have shown that the expression of the novel tumor microenvironment protein STC2 is negatively associated with survival in patients with several different types of human cancers, including gastric (Yokobori et al. 2010) and breast cancers (Hou et al. 2015) as well as renal cell carcinoma (Meyer et al. 2009). This suggests that STC2 is involved in tumor growth and metastasis. STC2 upregulation also appears to be associated with CRC progression and with the promotion of colon cancer cell migration (Ieta et al. 2009). STC2 plays an important role in the regulation of epithelial-mesenchymal transition (EMT) by suppressing several key regulators of EMT (Law and Wong 2010). In the present study, STC2 was upregulated in CRC-OxR cells, and the silencing of STC2 was beneficial for CRC-OxR sensitivity but had no effect on chemonaïve CRC cells. Our findings indicate that the hormone STC2 was secreted by 
CRC and became internalized to induce drug resistance in a previously unreported manner.

P-gp overexpression is considered to be the primary cause of MDR in oxaliplatin-resistant CRC (Ekblad et al. 2010). P-gp, an ATP-dependent membrane protein, is involved in the absorption, distribution, and excretion of drugs, xenobiotics, and endogenous compounds (Huls et al. 2009). In CRC, P-gp-mediated excretion of anticancer drugs from cells inhibits their cytotoxic effects (Hu et al. 2014). At present, research into MDR reversal in cancers is primarily aimed at identifying reversal agents that target P-gp. P-gp is upregulated via the activation of the NF- $\kappa \mathrm{B}$ signaling pathway (Liu et al. 2014). Glucosylceramide synthase upregulates P-gp (product of MDR1) expression to control anticancer drug resistance through the cSrc and $\beta$-catenin signaling pathways (Liu et al. 2010). A previous study has found that the transduction of HCT15 and HCT116 human colon carcinoma cell lines with human TNF and IL-2 reversed MDR (Stein et al. 1996). According to our present data, STC2 positively regulated P-gp expression and increased oxaliplatin efflux from HCT116 cells. Therefore, it can be suggested that the upregulation of the STC2/P-gp pathway is involved in the oxaliplatin-resistance mechanism of CRC cells.

PI3K/Akt activity has been suggested to play a regulatory role in the MDR of cancers (Abdul-Ghani et al. 2006). Certain drugs reverse MDR in cancers by inhibiting the PI3K/Akt signaling pathway (Choi et al. 2008; Cordo Russo et al. 2008). For instance, tamoxifen reverses P-gp-mediated MDR in gastric cancer cells by inhibiting the PI3K/Akt signaling pathway (Mao et al. 2014). Furthermore, PI3K/Akt 
pathway inhibition has been shown to block drug efflux from HT29RDB colon carcinoma cells (Abdul-Ghani et al. 2006). Consistent with previous findings, the present research shows that PI3K-shRNA and Akt-shRNA reversed the effects of STC2 on MDR in CRC. PI3K-shRNA and Akt-shRNA also inhibited STC2-induced P-gp expression. In the present study, we noted that the specific effect of STC2 on oxaliplatin resistance was mediated through a P-gp and PI3K/Akt signaling pathway-induced mechanism.

In conclusion, our study shows that STC2 overexpression is a critical component of the mechanism by which CRC cells become resistant to the cytotoxic effects of oxaliplatin. This resistance-promoting effect is mediated by PI3K/Akt and P-gp signaling. Our findings introduce significant translational potential for the identification of novel approaches to overcome STC2 dysregulation-related drug resistance. Moreover, STC2 expression could potentially be used as a predictive therapeutic biomarker for regimens utilizing oxaliplatin.

\section{References}

Abdul-Ghani, R., Serra, V., Gyorffy, B., Jurchott, K., Solf, A., Dietel, M., et al. 2006. The PI3K inhibitor LY294002 blocks drug export from resistant colon carcinoma cells overexpressing MRP1. Oncogene, 25(12):1743-1752.

Chang, A.C., Jellinek, D.A., and Reddel, R.R. 2003. Mammalian stanniocalcins and cancer. Endocr. Relat. Cancer, 10(3):359-373.

Choi, B.H., Kim, C.G., Lim, Y., Shin, S.Y., and Lee, Y.H. 2008. Curcumin 
down-regulates the multidrug-resistance mdr1b gene by inhibiting the PI3K/Akt/NF kappa B pathway. Cancer Lett. 259(1):111-118.

Comerford, K.M., Wallace, T.J., Karhausen, J., Louis, N.A., Montalto M.C., and Colgan, S.P. 2002. Hypoxia-inducible factor-1-dependent regulation of the multidrug resistance (MDR1) gene. Cancer Res. 62(12):3387-3394.

Cordo Russo, R.I., Garcia, M.G., Alaniz, L., Blanco, G., Alvarez, E., and Hajos, S.E. 2008. Hyaluronan oligosaccharides sensitize lymphoma resistant cell lines to vincristine by modulating P-glycoprotein activity and PI3K/Akt pathway. Int. J. Cancer, 122(5):1012-1018.

Davies, J.M., and Goldberg, R.M. 2008. First-line therapeutic strategies in metastatic colorectal cancer. Oncology (Williston Park), 22(13):1470-1479.

Ekblad, L., Kjellstrom, J., and Johnsson, A. 2010. Reduced drug accumulation is more important in acquired resistance against oxaliplatin than against cisplatin in isogenic colon cancer cells. Anticancer Drugs, 21(5):523-531.

Goldberg, R.M., Sargent, D.J., Morton, R.F., Fuchs, C.S., Ramanathan, R.K., Williamson S.K., et al. 2004. A randomized controlled trial of fluorouracil plus leucovorin, irinotecan, and oxaliplatin combinations in patients with previously untreated metastatic colorectal cancer. J. Clin. Oncol. 22(1):23-30.

Gottesman, M.M., and Ling, V. 2006. The molecular basis of multidrug resistance in cancer: the early years of P-glycoprotein research. FEBS Lett. 580(4):998-1009.

Hashemzadeh, S., Arabzadeh, A.A., Estiar, M.A., Sakhinia, M., Mesbahi, N., Emrahi L., et al. 2014. Clinical utility of measuring expression levels of Stanniocalcin 2 in 
patients with colorectal cancer. Med. Oncol. 31(10):237.

Hou, J., Wang, Z., Xu, H., Yang, L., Yu, X., Yang, Z., et al. 2015. Stanniocalicin 2 suppresses breast cancer cell migration and invasion via the PKC/claudin-1-mediated signaling. PloS One, 10(4):e0122179.

Hu, T., To, K.K., Wang, L., Zhang, L., Lu, L., Shen, J., et al. 2014. Reversal of P-glycoprotein (P-gp) mediated multidrug resistance in colon cancer cells by cryptotanshinone and dihydrotanshinone of Salvia miltiorrhiza. Phytomedicine, 21(11):1264-1272.

Huls, M., Russel, F.G., and Masereeuw, R. 2009. The role of ATP binding cassette transporters in tissue defense and organ regeneration. J. Pharmacol. Exp. Ther. 328(1):3-9.

Ieta, K., Tanaka, F., Yokobori, T., Kita, Y., Haraguchi, N., Mimori, K., et al. 2009. Clinicopathological significance of stanniocalcin 2 gene expression in colorectal cancer. Int. J. Cancer, 125(4):926-931.

Jepsen, M.R., Kloverpris, S., Mikkelsen, J.H., Pedersen, J.H., Fuchtbauer, E.M., Laursen L.S., et al 2015. Stanniocalcin-2 inhibits mammalian growth by proteolytic inhibition of the insulin-like growth factor axis. J. Biol. Chem. 290(6):3430-3439.

Jiang, B.H., and Liu L.Z. 2008. Role of mTOR in anticancer drug resistance: perspectives for improved drug treatment. Drug Resist. Updates, 11(3):63-76.

Kuan, C.Y., Walker, T.H., Luo, P.G., and Chen, C.F. 2011. Long-chain polyunsaturated fatty acids promote paclitaxel cytotoxicity via inhibition of the MDR1 gene in the human colon cancer Caco-2 cell line. J. Am. Coll. Nutr. 
30(4):265-273.

Kuo, T.C., Chiang, P.C., Yu, C.C., Nakagawa-Goto, K., Bastow, K.F., Lee, K.H., et al. 2011. A unique P-glycoprotein interacting agent displays anticancer activity against hepatocellular carcinoma through inhibition of GRP78 and mTOR pathways. Biochem. Pharmacol. 81(9):1136-1144.

Law, A.Y., and Wong, C.K. 2010. Stanniocalcin-2 promotes epithelial-mesenchymal transition and invasiveness in hypoxic human ovarian cancer cells. Exp. Cell Res. 316(20):3425-3434.

Law, A.Y., and Wong, C.K. 2013. Stanniocalcin-1 and -2 promote angiogenic sprouting in HUVECs via VEGF/VEGFR2 and angiopoietin signaling pathways. Mol. Cell Endocrinol. 374(1-2):73-81.

Liu, Y.Y., Gupta, V., Patwardhan, G.A., Bhinge, K., Zhao, Y., Bao, J., et al. 2010. Glucosylceramide synthase upregulates MDR1 expression in the regulation of cancer drug resistance through cSrc and beta-catenin signaling. Mol. Cancer, 9:145.

Liu, Z., Duan, Z.J., Chang, J.Y., Zhang, Z.F., Chu, R., Li, Y.L., et al. 2014. Sinomenine sensitizes multidrug-resistant colon cancer cells (Caco-2) to doxorubicin by downregulation of MDR-1 expression. PloS One, 9:e98560.

Loo, T.W., and Clarke, D.M. 2005. Recent progress in understanding the mechanism of P-glycoprotein-mediated drug efflux. J. Membr. Biol. 206(3):173-185.

Mao, Z., Zhou, J., Luan, J., Sheng, W., Shen, X., and Dong, X. 2014. Tamoxifen reduces $\mathrm{P}$-gp-mediated multidrug resistance via inhibiting the PI3K/Akt signaling pathway in ER-negative human gastric cancer cells. Biomed. Pharmacother. 
68(2):179-183.

Meyer, H.A., Tolle, A., Jung, M., Fritzsche, F.R., Haendler, B., Kristiansen, I., et al. 2009. Identification of stanniocalcin 2 as prognostic marker in renal cell carcinoma. Eur. Urol. 55(3):669-678.

Meyerhardt, J.A., and Mayer, R.J. 2005. Systemic therapy for colorectal cancer. N. Engl. J. Med. 352(5):476-487.

Murai, R., Tanaka, M., Takahashi, Y., Kuribayashi, K., Kobayashi, D., and Watanabe, N. 2014. Stanniocalcin-1 promotes metastasis in a human breast cancer cell line through activation of PI3K. Clin. Exp. Metastasis, 31(7):787-794.

Persons, D.L., Yazlovitskaya, E.M., Cui, W., and Pelling, J.C. 1999. Cisplatin-induced activation of mitogen-activated protein kinases in ovarian carcinoma cells: inhibition of extracellular signal-regulated kinase activity increases sensitivity to cisplatin. Clin. Cancer Res. 5(5):1007-1014.

Shin, J., and Sohn Y.C., 2014. Identification of Ran-binding protein $\mathrm{M}$ as a stanniocalcin 2 interacting protein and implications for androgen receptor activity. BMB Rep. 47:643-638.

Stein, U., Walther, W., and Shoemaker, R.H. 1996. Reversal of multidrug resistance by transduction of cytokine genes into human colon carcinoma cells. J. Natl. Cancer Inst. 88(19):1383-1392.

Szakacs, G., Paterson, J.K., Ludwig, J.A., Booth-Genthe, C., and Gottesman, M.M. 2006. Targeting multidrug resistance in cancer. Nat. Rev. Drug. Discov. 5(3):219-234.

Wagner, G.F., and Jaworski, E. 1994. Calcium regulates stanniocalcin mRNA levels in 
primary cultured rainbow trout corpuscles of stannius. Mol. Cell Endocrinol. 99(2):315-322.

Wagner, G.F., Jaworski, E.M., and Haddad, M. 1998. Stanniocalcin in the seawater salmon: structure, function, and regulation. Am. J. Physiol. 274:R1177-1185.

Wang, H., Zhao, L., Zhu, L.T., Wang, Y., Pan, D., Yao, J., et al. 2014. Wogonin reverses hypoxia resistance of human colon cancer HCT116 cells via downregulation of HIF-1alpha and glycolysis, by inhibiting PI3K/Akt signaling pathway. Mol. Carcinog. 53 Suppl 1:E107-118.

Wang, X., Martindale, J.L., and Holbrook, N.J. 2000. Requirement for ERK activation in cisplatin-induced apoptosis. J. Biol. Chem. 275(50):39435-39443.

Wang, Z., Zhang, L., Ni, Z., Sun, J., Gao, H., Cheng, Z., et al. 2015. Resveratrol induces AMPK-dependent MDR1 inhibition in colorectal cancer HCT116/L-OHP cells by preventing activation of NF-kappaB signaling and suppressing cAMP-responsive element transcriptional activity. Tumour Biol. (in press).

Yang, A.D., Fan, F., Camp, E.R., van Buren, G., Liu, W., Somcio, R., et al. 2006. Chronic oxaliplatin resistance induces epithelial-to-mesenchymal transition in colorectal cancer cell lines. Clin. Cancer Res. 12(14 Pt 1):4147-4153.

Yokobori, T., Mimori, K., Ishii, H., Iwatsuki, M., Tanaka, F., Kamohara, Y., et al. 2010. Clinical significance of stanniocalcin 2 as a prognostic marker in gastric cancer. Ann. Surg. Oncol. 17(10):2601-2607.

Zeng, X., Yang, P., Chen, B., Jin, X., Liu, Y., Zhao, X., et al. 2013. Quantitative secretome analysis reveals the interactions between epithelia and tumor cells by in 
vitro modulating colon cancer microenvironment. J. Proteomics, 89:51-70. 


\section{Figure Legends}

Fig. 1. STC2 expression in oxaliplatin-resistant (OxR) CRC cells. A: Compared with the expression levels in parental (PAR) cell lines, STC2 protein expression was upregulated in CRC-OxR cells, as detected by Western blot analysis. B: STC2 concentrations were increased in the supernatants of CRC-OxR cells compared with those in PAR cell lines, as detected by an enzyme-linked immunosorbent assay ${ }^{* *} P<$ 0.01 vs. PAR. Results are representative of three independent experiments.

Fig. 2. STC2-shRNA reversed oxaliplatin (Oxali) resistance in HCT116-OxR cells A: The STC2 protein expression level decreased significantly following transient transfection of HCT116-OxR cells with STC2-shRNA, as measured by Western blot analysis. B: STC2 secreted in the medium was decreased by STC2-shRNA transfection. C: The representative images of apoptosis, as detected by annexin V and propidium iodide staining. D: STC2-shRNA re-sensitized HCT116-OxR cells to Oxali at $48 \mathrm{~h}$ post-transfection, as demonstrated by the induction of apoptosis. E: STC2-shRNA enhanced the effect of Oxali on caspase-3 activity in HCT116-OxR cells. ${ }^{*} P<0.05$ and ${ }^{* *} P<0.01$ vs. N-shRNA. Results are representative of three independent experiments. 
Fig. 3. STC2-induced oxaliplatin (Oxali) resistance in HCT116 cells A: STC2 (10 $\mathrm{pg} / \mathrm{ml}$ ) inhibited the apoptosis-inducing effect of Oxali at $48 \mathrm{~h}$ post-transfection, as detected by annexin $\mathrm{V}$ and propidium iodide staining. B: STC2 (10 pg/ml) also inhibited Oxali-induced caspase-3 activation. ${ }^{*} P<0.05$ and ${ }^{* *} P<0.01$ vs. control. Results are representative of three independent experiments.

Fig. 4. P-gp expression in colorectal cancer (CRC)-OxR cells A: Compared with PAR cells, P-gp was upregulated in CRC-OxR cells, as detected by Western blot analysis. B: STC2-shRNA downregulated P-gp protein expression in the HCT116-OxR cell line. C: STC2-shRNA inhibited drug efflux in the HCT116-OxR cell line, as detected by rhodamine-123 transport. D: Transepithelial transport of rhodamine-123 in the apical (A)-to-basolateral (B) direction (right) across HCT116-OxR cell monolayers in the control (०), N-shRNA- (•), and STC2-shRNA-transfected cells $(\boldsymbol{\nabla}) .{ }^{* *} \mathrm{P}<0.01$ vs. PAR; ${ }^{+} \mathrm{P}<0.05,{ }^{++} \mathrm{P}<0.01$ vs. N-shRNA. Results are representative of three independent experiments.

Fig. 5. Role of the PI3K/Akt signaling pathway in STC2-induced CRC-OxR cell

lines A: PI3K and p-AKT were downregulated by STC2-shRNA in HCT116-OxR, HT-29-OxR, and RKO-OxR cell lines. B: PI3K-shRNA and AKT-shRNA inhibited P-gp expression in the HCT116-OxR cell line. C: PI3K-shRNA and AKT-shRNA 
increased the concentration of rhodamine-123 efflux in the HCT116-OxR cell line. D:

Transepithelial transport of rhodamine-123 in the apical (A)-to-basolateral (B) direction (right) across HCT116-OxR cell monolayers in control (०), N-shRNA- (•), PI3K-shRNA- $(\boldsymbol{\nabla})$, and AKT-shRNA-transfected cells $(\triangle) .{ }^{*} \mathrm{P}<0.05,{ }^{* *} \mathrm{P}<0.01$ vs. $\mathrm{N}$-siRNA. Results are representative of three independent experiments.

Fig. S1. STC2-shRNA did not increase the sensitivity of chemonaïve HCT116 cells to oxaliplatin (Oxali) A: The STC2 protein expression level decreased significantly following the transient transfection of chemonaïve HCT116 cells with STC2-shRNA, as measured by Western blotting. B: STC2 secreted in the medium decreased by the transfection of the cells with STC2-shRNA. C: The effect of STC2-shRNA on apoptosis in HCT116 cells treated with Oxali $48 \mathrm{~h}$ post-transfection.

D: The effect of STC2-shRNA on caspase-3 activity in HCT116 cells treated with Oxali. ${ }^{*} P<0.05$ and ${ }^{* *} P<0.01$ vs. N-shRNA. Results are representative of three independent experiments. 

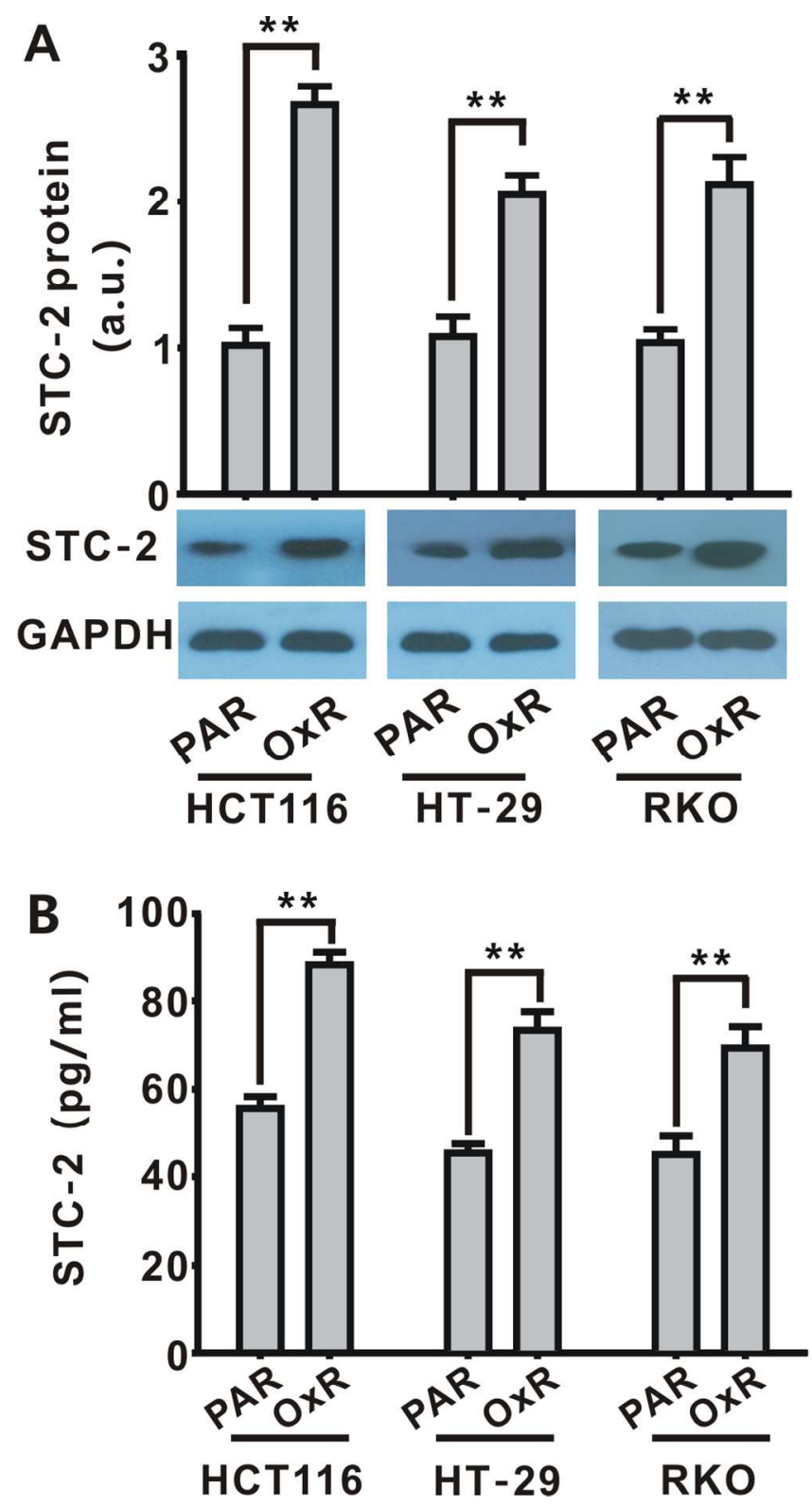

Fig. 1. STC2 expression in oxaliplatin-resistant (OxR) CRC cells. A: Compared with the expression levels in parental (PAR) cell lines, STC2 protein expression was upregulated in CRC-OxR cells, as detected by Western blot analysis. B: STC2 concentrations were increased in the supernatants of CRC-OxR cells compared with those in PAR cell lines, as detected by an enzyme-linked immunosorbent assay $* * P<0.01$ vs. PAR. Results are representative of three independent experiments. 


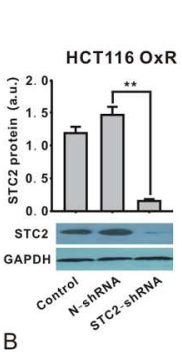

B

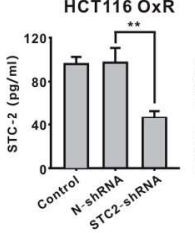

C
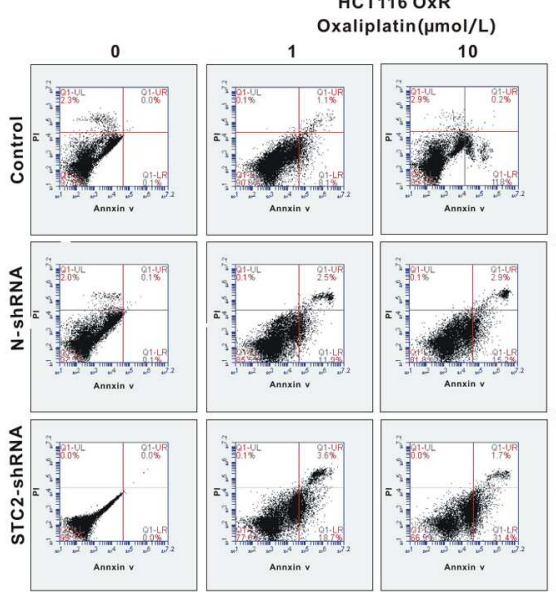

50
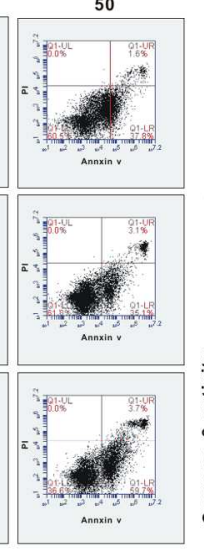

D

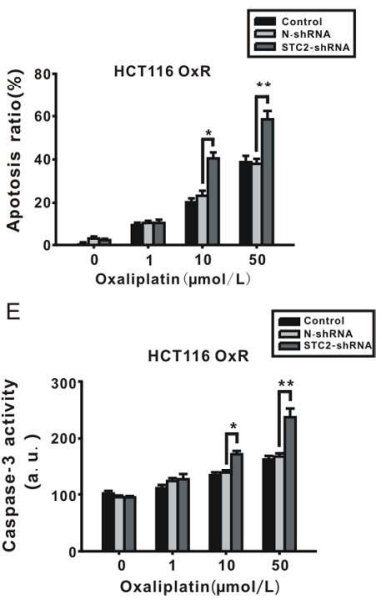

Fig. 2. STC2-shRNA reversed oxaliplatin (Oxali) resistance in HCT116-OxR cells A: The STC2 protein expression level decreased significantly following transient transfection of HCT116-OxR cells with STC2shRNA, as measured by Western blot analysis. B: STC2 secreted in the medium was decreased by STC2shRNA transfection. C: The representative images of apoptosis, as detected by annexin $\mathrm{V}$ and propidium iodide staining. D: STC2-shRNA re-sensitized HCT116-OxR cells to Oxali at $48 \mathrm{~h}$ post-transfection, as demonstrated by the induction of apoptosis. E: STC2-shRNA enhanced the effect of Oxali on caspase-3 activity in HCT116-OxR cells. ${ }^{*} \mathrm{P}<0.05$ and $* * \mathrm{P}<0.01 \mathrm{vs}$. N-shRNA. Results are representative of three independent experiments. 
A
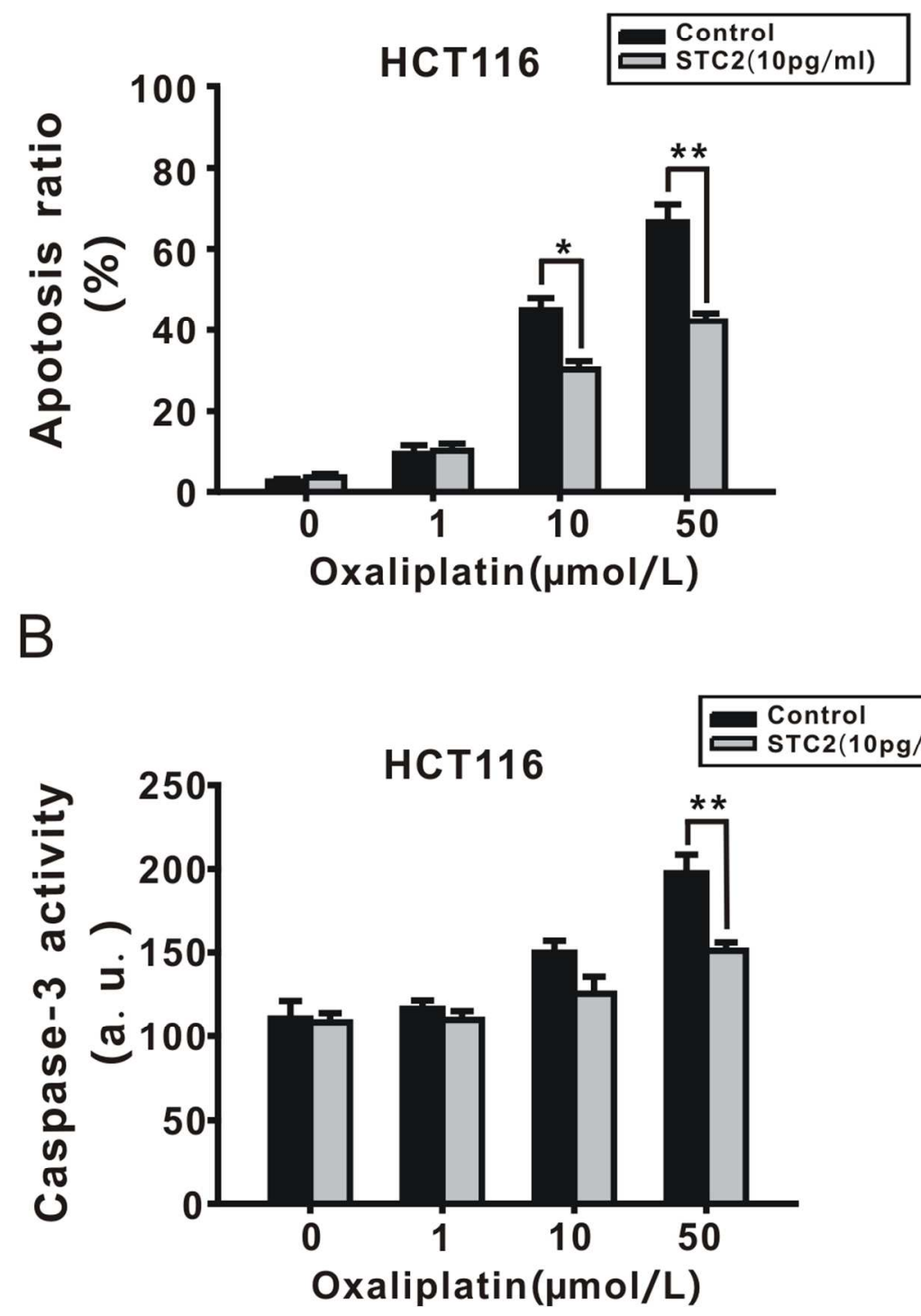

Fig. 3. STC2-induced oxaliplatin (Oxali) resistance in HCT116 cells A: STC2 $(10 \mathrm{pg} / \mathrm{ml})$ inhibited the apoptosis-inducing effect of Oxali at $48 \mathrm{~h}$ post-transfection, as detected by annexin $\mathrm{V}$ and propidium iodide staining. B: STC2 $(10 \mathrm{pg} / \mathrm{ml})$ also inhibited Oxali-induced caspase-3 activation. $* \mathrm{P}<0.05$ and $* * \mathrm{P}<0.01$ vs. control. Results are representative of three independent experiments. 

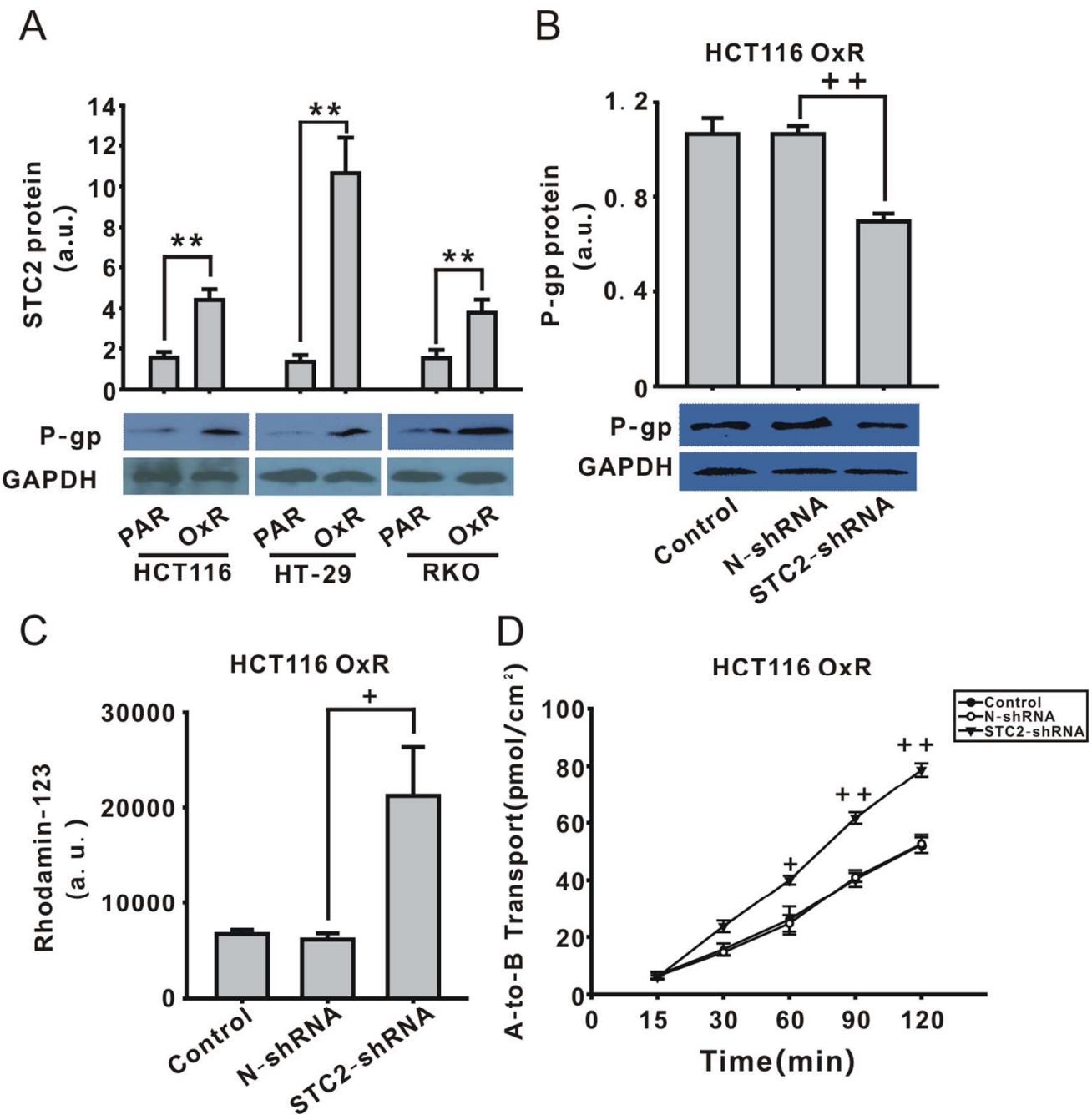

Fig. 4. P-gp expression in colorectal cancer (CRC)-OxR cells A: Compared with PAR cells, P-gp was upregulated in CRC-OxR cells, as detected by Western blot analysis. B: STC2-shRNA downregulated P-gp protein expression in the HCT116-OxR cell line. C: STC2-shRNA inhibited drug efflux in the HCT116-OxR cell line, as detected by rhodamine-123 transport. D: Transepithelial transport of rhodamine-123 in the apical (A)-to-basolateral (B) direction (right) across HCT116-OxR cell monolayers in the control (0), N-shRNA- (•), and STC2-shRNA-transfected cells $(\boldsymbol{\nabla})$. ${ }^{* * P}<0.01$ vs. PAR; $+\mathrm{P}<0.05,++\mathrm{P}<0.01$ vs. N-shRNA. Results are representative of three independent experiments. 
A
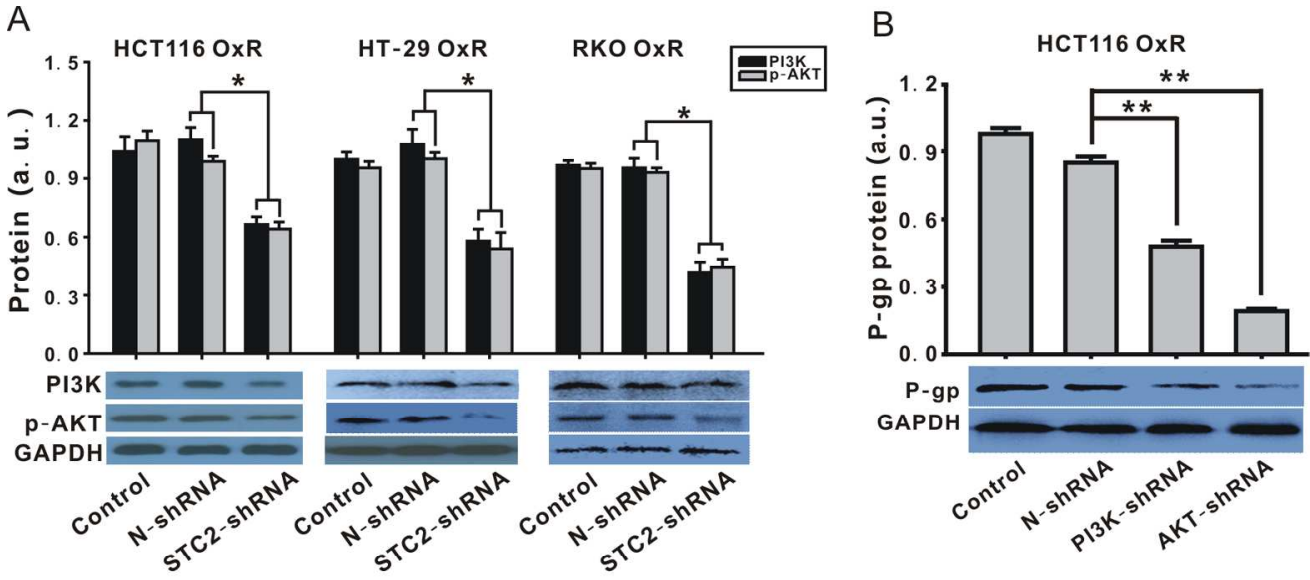

C

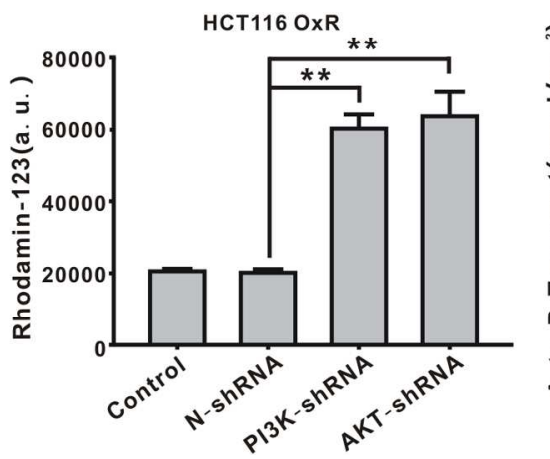

D
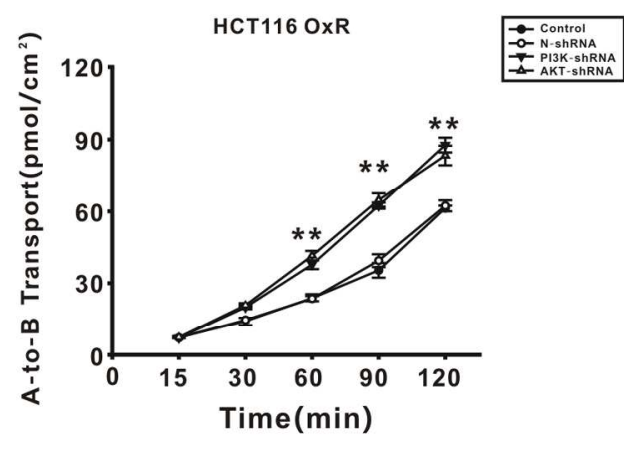

PISK-shRNA
$-\mathrm{AKT}-$-shRNA

Fig. 5. Role of the PI3K/Akt signaling pathway in STC2-induced CRC-OxR cell lines A: PI3K and p-AKT were downregulated by STC2-shRNA in HCT116-OxR, HT-29-OxR, and RKO-OxR cell lines. B: PI3K-shRNA and AKT-shRNA inhibited P-gp expression in the HCT116-OxR cell line. C: PI3K-shRNA and AKT-shRNA increased the concentration of rhodamine-123 efflux in the HCT116-OxR cell line. D: Transepithelial transport of rhodamine-123 in the apical (A)-to-basolateral (B) direction (right) across HCT116-OxR cell monolayers in control $(0)$, N-shRNA- $(\bullet)$, PI3K-shRNA- $(\boldsymbol{\nabla})$, and AKT-shRNA-transfected cells $(\Delta)$. *P $<0.05, * * P<0.01$ vs. N-siRNA. Results are representative of three independent experiments. 